\title{
Book

\section{Handbook of Dissolution Testing, 3rd edition Royal Hanson and Vivian Gray, published by Dissolution Technologies, Inc., 2004 ISBN 0-9761519-0-1}

e-mail:hbrittain@earthlink.net

\author{
Harry G. Brittain, Ph.D. \\ Center for Pharmaceutical Physics, 10 Charles Road, Milford, NJ 08848
}

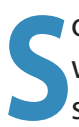
o, you have just received the assignment of becoming a working expert in the field of dissolution testing. That should not be too difficult if you have been working in the area for a while, but what does an analyst do if he or she suddenly has to gain a comprehensive knowledge of the field in a short time? There might be other options that are beyond the scope of this review, but the most advisable course of action would be to obtain a copy of the latest edition of the Handbook and to read it from cover to cover. Given the degree of coverage in this third edition, the time would be well spent.

The Handbook consists of 8 chapters and an appendix, and begins with a general discussion as to why one would want to conduct dissolution testing (aside from the reason given above). The chapter opens with a bit of history, discussing disintegration testing and how this methodology became replaced by prototype and official dissolution test methods. The subjects of dissolution testing standards and calibrators are broached, but then the chapter gets into the real heart of the subject, summarizing the characteristics of various methods of dissolution testing. Capsule summaries of the basket, paddle, and flow-through methods are provided, as well as discussions of the reciprocating cylinder apparatus and transdermal dissolution testing.

The second chapter briefly reviews some theoretical aspects of the topic, but this coverage is not extensive, and interested readers would need to consult the literature for more detail if they were so concerned. The concept of dissolution rate is defined and is followed by a good discussion of the surface area of a dissolving solid and its effect on flow dynamics. The last topic of import concerns bioequivalence and its relation to dissolution characteristics, but that is a complicated question and really only outlined in this presentation.

Most workers will pay special attention to the third chapter, namely the dissolution testing of solid dose forms. Discussed in this chapter are the basket system (USP Apparatus 1 ), the paddle system (USP Apparatus 2), the recipro- cating cylinder (USP Apparatus 3), and the flow-through cell (USP Apparatus 4). Specifications for all four apparatus types are presented, and there is a most useful discussion of the compendial constraints that must be considered when using the two most popular dissolution methods, namely the paddle and the basket.

However, not every analyst is directed to test only solid dose products, and hence chapter 4 covers the dissolution testing of other drug delivery forms. The use of flowthrough cells, paddle-over-disk arrangements, rotating cylinders, reciprocating holders, vertical diffusion cells, ointment cells, and suppository baskets is covered in this chapter. Recommendations for the testing of special dosage forms, such as oral suspensions, orally disintegrating tablets, chewable tablets, transdermal patches, topicals, suppositories, chewing gums, powders, granules, dispersions, and parenterals, are also provided.

Every mode of analytical testing requires the setting of operational parameters that ensure that a validated method can be reliably used. Chapter 5 discusses what variables need to be controlled. Regarding Apparatus 1 and 2, the eccentricity of the stirring drive shaft and its straightness are discussed, as well as other stirring device variables. The important topic of environmental vibration and its effect on the system is discussed. Obviously, one needs to consider the properties of the dissolution medium itself, so the topics of gases and the need for deaeration, $\mathrm{pH}$, fluid volume, temperature, the sink condition, and vessel hydrodynamics are discussed. This chapter also contains a very useful checklist for experimental variables, anticipated degrees of variation, and methods for their control that will greatly assist during ruggedness testing of a dissolution method.

Sooner or later, it is inevitable that an analyst has to go into the laboratory and actually conduct some experimental work. Chapter 6 counsels as how to go about setting up a system for dissolution testing, and here the topics of dissolution protocols, inspection of paddles and shafts, checking eccentricity (of the system, not the analyst), speed control, and vibration are covered. The use of filtration methods, 
calibration of equipment, the use of calibrator tablets, and compendial calibration requirements are discussed as well. In my opinion, chapter 7 could be the most important of all in that it discusses some commonly encountered practical aspects (poorly soluble drugs; low analyte concentrations; difficult dosage forms; dosage composition, size, and type; floating dosage forms; and change in $\mathrm{pH}$ during testing) as well as method development and validation. For the latter, readers will find a checklist for method development, advice on selection of methods for various drug entitles, and an overview of method validation as applied to dissolution testing.

The concluding chapter 8 concerns the automation of dissolution testing as a means of improving through-put and avoiding analyst saturation. There is sufficient discussion regarding how to define the work flow to design the automation process and how to deal with data handling questions. This chapter is followed by an appendix that summarizes a working validation guideline, and then moves on to Installation Qualification, Operational Qualification, and Performance Qualification.

This book makes a highly useful addition to the sufficient body of knowledge outlined in USP general tests $<711>$ and $<724>$. Topics are discussed in just enough depth for a busy analyst to learn what needs to be learned in a short period of time and to then get back to the bench for a continuation of productive work. Those analysts needing more detail than has been provided in this book will undoubtedly consult the leading works in the field, and that is just the way of things. However, it is clear that the information contained in the third edition of the Handbook will fulfill important needs and should therefore be on the shelf of every analyst either contemplating or performing dissolution testing. 